\title{
The method of finding solutions of partial dynamic equations on time scales
}

\section{Hsuan-Ku Liu*}

\section{"Correspondence:}

HKLiu.nccu@gmail.com

Department of Mathematics and

Information Education, National

Taipei University of Education,

Taipei, Taiwan

\begin{abstract}
On time scales, one area lacking of development is the method of finding solutions on partial dynamic equations. This paper proposes a method for finding the exact solution of linear partial dynamic equations on arbitrage time scales. We modify the variational iteration method on $\mathbb{R}$ to find an approximation of the nonlinear partial dynamic equation on $\overline{q^{\mathbb{N}}}$. As an example, the modified variational iteration method is applied to $q$-Berger equations and to $q$-Fisher equations. Their numerical results reveal that the proposed method is very effective.
\end{abstract}

Keywords: partial dynamic equations on time scales; nonlinear q-difference equation; variational iterative method; approximate solutions

\section{Introduction}

A time scale is a nonempty closed subset of real numbers. On time scale calculus, notations and theorems have been well established for the univariate case [1]. Solutions of ordinary differential equations, such as initial value problems and boundary value problems, have been studied and published during the past two decades on time scales. In recent years, Hoffacker [2] and Ahlbrandt and Morian [3] demonstrated the related ideas to the multivariate case and studied partial dynamic equations on time scales. Notations and definitions on multivariate time scales calculus can be found in Bohner and Guseinov $[4,5]$. Jackson [6] extended the existing ideas of the time scales calculus [3] to the multivariate case. The method of generalized Laplace transform on time scales is applied to find solutions of the homogeneous and nonhomogeneous heat and wave equations. Recent developments in the method of finding solutions have aroused further interest in the discussion of partial dynamic equations on time scales.

For the nonlinear cases, methods of finding solutions are not mentioned for partial dynamic equations on time scales. One of the difficulties for developing a theory of series solutions to nonlinear equations on time scales is that the formula for multiplications of two generalized polynomials is not easily found. If a time scale has constant graininess, Haile and Hall [7] provided an exact formula for the multiplication of two generalized polynomials. Using the obtained results, the series solutions for linear dynamic equations are proposed on the time scales $\mathbb{R}$ and $\mathbb{T}=h \mathbb{Z}$ (difference equations with step size $h$ ). On generalized time scales, Mozyrska and Pawtuszewicz [8] presented a formula for the multiplication of generalized polynomials of degree one and degree $n \in \mathbb{N}$. Liu [9] provided a product rule of two generalized polynomials on the time scale $\overline{q^{\mathbb{Z}}}=\left\{q^{n} \mid n \in \mathbb{N}\right\} \cup\{0\}$.

2013 Liu; licensee Springer. This is an Open Access article distributed under the terms of the Creative Commons Attribution License (http://creativecommons.org/licenses/by/2.0), which permits unrestricted use, distribution, and reproduction in any medium, provided the original work is properly cited. 
The variational iteration method proposed by $\mathrm{He}[10]$ is a powerful mathematical tool in analyzing the nonlinear problems on $\mathbb{R}$ (the set of real numbers). Over the last few years, the variational iteration method (VIM) has been widely applied to analyze the nonlinear boundary value problems [11], the nonlinear heat diffusion equations [12] and the nonlinear reaction-diffusion equations [13]. An advantage of the VIM is that there is no need to make the assumption of the small parameters. On nonlinear partial dynamic equations, approximate solutions obtained by the variational iteration method are not found yet.

In this paper, we first explore a simple method to find the exact solution of linear partial dynamic equations on time scales. For the nonlinear cases, we derive a product rule of two generalized polynomials on $\overline{q^{\mathbb{Z}}}$, which provides an idea for developing a series solutions on $q$-calculus. Applying the product rules, we extend the variational iteration method from the set of real numbers $\mathbb{R}$ to the time scales $\overline{q^{\mathbb{Z}}}$. The extension provides a method to find an approximate solution on the nonlinear partial dynamic equation on $\overline{q^{\mathbb{Z}}}$. Moreover, the VIM is applied to find an approximation of the $q$-Berger equation and the $q$-Fisher equation. By the numerical results, we found that the modified VIM is very effective. The VIM can be applied to other time scales when the multiplication rule of two generalized polynomials on these time scales is obtained.

This paper is organized as follows. In Section 2, the basic ideas of partial dynamic equations on time scales are introduced. In Section 3, a method is explored to find an exact solution of linear initial value problems on time scales. In Section 4, a product rule of two generalized polynomials at 0 is derived on $\overline{q^{\mathbb{Z}}}$ and the variational iteration method is applied to find an approximate solution of the Burger equation and the Fisher equation. In Section 5, numerical examples reveal that the proposed method is very effective. Finally, a concise conclusion and future directions are provided in Section 6.

\section{Basic concepts on time scales}

A time scale is an arbitrary nonempty closed subset of the real numbers. The calculus of time scales was introduced by Hilger [14] in order to create a theory that can unify discrete and continuous analysis.

\subsection{An introduction to time scales}

In this subsection, we first define the forward and backward jump operators on time scales and then introduce the delta derivative and the integration.

Definition 1 Let $\mathbb{T}$ be a time scale. For $t \in \mathbb{T}$ the forward jump operator $\sigma: \mathbb{T} \rightarrow \mathbb{T}$ is defined by

$$
\sigma(t):=\inf \{s>t \mid s \in \mathbb{T}\}
$$

and the backward jump operator $\rho: \mathbb{T} \rightarrow \mathbb{T}$ is defined by

$$
\rho(t):=\sup \{s<t \mid s \in \mathbb{T}\} .
$$

The gain function $\mu: \mathbb{T} \rightarrow[0, \infty)$ is defined by

$$
\mu(t):=\sigma(t)-t
$$


According to the forward jump operator and the gain function, the delta derivative on the time scale $\mathbb{T}$ is given as follows.

Definition 2 Assume that $f: \mathbb{T} \rightarrow \mathbb{R}$ is a function and let $t \in \mathbb{T}$. If $\sigma(t)>t$, the delta derivative of $f(t)$ at $t$ on the time scale $\mathbb{T}$ is defined as

$$
f^{\Delta}(t)=\frac{f(\sigma(t))-f(t)}{\mu(t)} .
$$

A function $f(t)$ on $\mathbb{T}$ is said to be differentiable at $t$ if its derivative exists at $t, \forall t \in \mathbb{T}$.

Integration on a time scale can be viewed as an anti-derivative.

Definition 3 If we have delta derivative $g(t)=f^{\Delta}(t)$ on the time scale $\mathbb{T}$, then the antiderivative is

$$
f(t)=\int_{a}^{t} g(s) \Delta s+\text { constant }, \quad a, t \in \mathbb{T}
$$

and the definite integral on the time scale $\mathbb{T}$ follows as

$$
\int_{a}^{b} g(s) \Delta s=f(b)-f(a), \quad a, b \in \mathbb{T}
$$

Following the delta derivative and integration, we define the generalized polynomials as follows.

Definition 4 On the time scale $\mathbb{T}$, the generalized polynomials $h_{k}\left(\cdot, t_{0}\right): \mathbb{T} \rightarrow \mathbb{R}$ are defined recursively as follows:

$$
h_{0}(t, s)=1, \quad h_{k+1}(t, s)=\int_{s}^{t} h_{k}(\tau, s) \Delta \tau .
$$

Hence, for each fixed $s$, the delta derivative of $h_{k}$ with respect to $t$ satisfies

$$
h_{k}^{\Delta j}(t, 0)= \begin{cases}h_{k-j}(t, 0) & \text { if } k \geq j, \\ 0 & \text { if } k<j .\end{cases}
$$

\subsection{An introduction to $q$-calculus}

Let

$$
q^{\mathbb{N}}=\left\{q^{n} \mid n \in \mathbb{N}\right\} \quad \text { and } \quad \overline{q^{\mathbb{N}}}=q^{\mathbb{N}} \cup\{0\}
$$

where $\mathbb{N}$ denotes the set of positive integers.

If $a$ and $q$ are real numbers such that $0<q<1$, then the $q$-shift factorial [15] is defined by

$$
(a ; q)_{0}=1 \quad \text { and } \quad(a ; q)_{n}=\prod_{k=0}^{n-1}\left(1-a q^{k}\right), \quad n \in \mathbb{N} .
$$

Following Definition 2, the $q$-derivative is given as follows. 
Definition 5 Assume that $f: \overline{q^{\mathbb{N}}} \rightarrow \mathbb{R}$ is a function and $t \in \overline{q^{\mathbb{N}}}$. The $q$-derivative [1] at $t$ is defined by

$$
f^{\Delta}(t)=\frac{f(q t)-f(t)}{(q-1) t}, \quad t \neq 0
$$

and

$$
f^{\Delta}(0)=\lim _{n \rightarrow \infty} \frac{f\left(q^{n}\right)-f(0)}{q^{n}} .
$$

By computing the recurrence relation, the $q$-polynomials are represented as

$$
h_{k}(t, s)=\prod_{v=0}^{k-1} \frac{t-s q^{v}}{\sum_{j=0}^{v} q^{j}}
$$

on $\overline{q^{\mathbb{N}}}[1]$.

A $q$-difference equation is an equation that contains $q$-derivatives of a function defined on $\overline{q^{\mathbb{N}}}$.

\subsection{Multivariable calculus on time scales}

The differentiation and integrations are introduced for functions of two variables on time scales [3]. Definitions on multivariate calculus on time scales can be found in Bohner and Guseinov [4,5]. Following the line of ideas, the dynamic equations on time scales are extended to partial dynamic equations on time scales [2, 3, 14].

Let $\mathbb{T}_{1}$ and $\mathbb{T}_{2}$ be any two time scales. Consider the 'rectangle' $\mathbb{T}=\mathbb{T}_{1} \times \mathbb{T}_{2}$. For any $\mathbf{t} \in \mathbb{T}$, the jump operator of $\mathbf{t}=\left(t_{1}, t_{2}\right)$ for $t_{1} \in \mathbb{T}_{1}$ and $t_{2} \in \mathbb{T}_{2}$ is given as follows:

1. The forward jump operators $\sigma: \mathbb{T} \rightarrow \mathbb{T}$ by $\sigma(\mathbf{t})=\left(\sigma\left(t_{1}\right), \sigma\left(t_{2}\right)\right)$ are defined as $\sigma\left(t_{1}\right)=\inf \left(s \in \mathbb{T}_{1} \mid s>t_{1}\right)$ and $\sigma\left(t_{2}\right)=\inf \left(s \in \mathbb{T}_{2} \mid s>t_{2}\right)$.

2. The backward jump operators $\tau: \mathbb{T} \rightarrow \mathbb{T}$ by $\tau(\mathbf{t})=\left(\tau\left(t_{1}\right), \tau\left(t_{2}\right)\right)$ are defined as $\tau\left(t_{1}\right)=\sup \left(s \in \mathbb{T}_{1} \mid s<t_{1}\right)$ and $\tau\left(t_{2}\right)=\sup \left(s \in \mathbb{T}_{2} \mid s<t_{2}\right)$.

To use the notations for partial derivatives with respect to time scale variables $t_{1}$ and $t_{2}$, respectively, we employ lexicographic ordering for consistency. Let $f^{\Delta_{1}}$ denote the time scale partial derivative with respect to $t_{1}$ and let $f^{\Delta_{2}}$ denote the time scale partial derivative with respect to $t_{2}$. Definitions of these partial derivatives are given as below $[2,3]$.

Definition 6 Let $f$ be a real-valued function on $\mathbb{T}$. At $\left(t_{1}, t_{2}\right) \in \mathbb{T}=\mathbb{T}_{1} \times \mathbb{T}_{2}$ we say $f$ has a $\Delta_{1}$-partial derivative $f^{\Delta_{1}}\left(t_{1}, t_{2}\right)$ if for each $\varepsilon>0$, there exists a neighborhood $U$ of $t_{1}$, with $U=\left(t_{1}-\delta, t_{1}+\delta\right) \cap \mathbb{T}_{1}$ for $\delta>0$, such that

$$
\left|f\left(\sigma\left(t_{1}\right), t_{2}\right)-f\left(s, t_{2}\right)-f^{\Delta_{1}}\left(t_{1}, t_{2}\right)\left(\sigma\left(t_{1}\right)-s\right)\right| \leq\left|\sigma\left(t_{1}\right)-s\right|
$$

for all $s \in U$. On the other hand, we say $f$ has a $\Delta_{2}$-partial derivative $f^{\Delta_{2}}\left(t_{1}, t_{2}\right)$ if for each $\varepsilon>0$, there exists a neighborhood $V$ of $t_{2}$, with $V=\left(t_{2}-\delta, t_{2}+\delta\right) \cap \mathbb{T}_{2}$ for $\delta>0$, such that

$$
\left|f\left(t_{1}, \sigma\left(t_{2}\right)\right)-f\left(t_{1}, s\right)-f^{\Delta_{2}}\left(t_{1}, t_{2}\right)\left(\sigma\left(t_{2}\right)-s\right)\right| \leq\left|\sigma\left(t_{2}\right)-s\right|
$$

for all $s \in V$. 
Using the ideas of time scale partial derivatives, notations of mixed partial and high order partial derivatives are given as follows:

1. $f^{\Delta_{i j}}(\mathbf{t})$ (if this value exists) denotes first taking the partial derivative with respect to $t_{i}$ and then taking the partial derivative with respect to $t_{j}$, so that $f^{\Delta_{i j}}=\left(f^{\Delta_{i}}\right)^{\Delta_{j}}$, $i, j=1,2$.

2. $f^{\Delta_{i}^{n}}(\mathbf{t})$ (if this value exists) denotes taking the partial derivative of $f(\mathbf{t})$ with respect to $t_{i} n$ times.

The details and examples can be found in [3] and [6].

\section{The exact solution of linear initial value problems on time scales}

Let $h_{k}\left(t_{1}, 0\right)$ and $g_{k}\left(t_{2}, 0\right)$ be the generalized polynomials on $\mathbb{T}_{1}$ and $\mathbb{T}_{2}$, respectively. In this section, the variational iteration method on $\mathbb{R}$ is extended to provide a method of finding the exact solution of linear partial dynamic equations on time scales. The introduction and the details of the variational iteration method can be found in the Appendix and in [10].

\subsection{The exact solution of the first-order linear partial dynamic equations}

We first consider the first-order linear partial dynamic equations as the form

$$
\left\{\begin{array}{l}
u^{\Delta_{1}}=c^{2} u^{\Delta_{2}} \quad \text { on } \mathbb{T}_{1} \times \mathbb{T}_{2}, \\
u\left(0, t_{2}\right)=f\left(t_{2}\right) \quad \text { on } \mathbb{T}_{2},
\end{array}\right.
$$

where $f\left(t_{2}\right)=\sum_{i=0}^{K} a_{i} g_{i}\left(t_{2}, 0\right)$ on $\mathbb{T}_{2}$ and $a_{i}, i=1, \ldots, K$ are real numbers.

The basic character of the variational iteration method is to construct a correction functional for the system, which reads

$$
u_{n+1}\left(t_{1}, t_{2}\right)=u_{n}\left(t_{1}, t_{2}\right)+\int_{t_{0}}^{t_{1}} \lambda\left\{L u_{n}\left(s, t_{2}\right)+N \tilde{u}_{n}\left(s, t_{2}\right)\right\} \Delta s,
$$

where $L$ is a linear operator on $\mathbb{T}_{1}, N$ is a linear (or nonlinear) operator on $\mathbb{T}_{2}\left(\right.$ or $\mathbb{T}_{1} \times \mathbb{T}_{2}$ ), $\lambda$ is a Lagrange multiplier which can be identified optimally by variational theory, $u_{n}$ is the $n$th approximation, and $\tilde{u}_{n}$ denotes a restricted variation, that is, $\delta \tilde{u}_{n}=0$.

The linear operator $L$ is selected as

$$
L u=u^{\Delta_{1}}
$$

and the other operator $N$ is selected as

$$
N u=-c^{2} u^{\Delta_{2}} .
$$

Make the above correction functional stationary with respect to $u_{n}$

$$
\begin{aligned}
\delta u_{n+1}\left(t_{1}, t_{2}\right) & =\delta u_{n}\left(t_{1}, t_{2}\right)+\delta \int_{0}^{t_{1}} \lambda\left\{u^{\Delta_{1}}\left(s, t_{2}\right)+N \tilde{u}_{n}\left(s, t_{2}\right)\right\} \Delta s \\
& =\left(1+\lambda\left(t_{1}\right)\right) \delta u_{n}\left(t_{1}, t_{2}\right)+\int_{0}^{t_{1}} \lambda^{\Delta}(s) \delta u_{n}\left(\sigma(s), t_{2}\right) \Delta s .
\end{aligned}
$$


We, therefore, have the following stationary conditions:

$$
\begin{aligned}
& 1+\lambda\left(t_{1}\right)=0, \\
& \lambda^{\Delta}(s)=0 .
\end{aligned}
$$

The Lagrange multiplier can be readily identified

$$
\lambda(s)=-1 .
$$

As a result, the variational iteration formula is obtained

$$
u_{n+1}\left(t_{1}, t_{2}\right)=u_{n}\left(t_{1}, t_{2}\right)-\int_{0}^{t}\left\{u_{n}^{\Delta_{1}}\left(s, t_{2}\right)+N u_{n}\left(s, t_{2}\right)\right\} \Delta s .
$$

Using the initial condition $u_{0}=f\left(t_{2}\right)$ and the iteration formula (2), we have the following equations:

$$
\begin{aligned}
u_{1}\left(t_{1}, t_{2}\right) & =f\left(t_{2}\right)-\int_{0}^{t_{1}} f^{\Delta_{2}}\left(t_{2}\right) \Delta s \\
& =f\left(t_{2}\right)+c^{2} f^{\Delta_{2}}\left(t_{2}\right) h_{1}\left(t_{1}, 0\right), \\
u_{2}\left(t_{1}, t_{2}\right) & =u\left(t_{1}, t_{2}\right)-\int_{0}^{t_{1}} c^{2} f^{\Delta_{2}^{2}}\left(t_{2}\right) h_{1}(s, 0) \Delta s \\
& =u_{1}\left(t_{1}, t_{2}\right)+c^{4} f^{\Delta_{2}^{2}}\left(t_{2}\right) h_{2}\left(t_{1}, 0\right) \\
& =f\left(t_{2}\right)+c^{2} f^{\Delta_{2}}\left(t_{2}\right) h_{1}\left(t_{1}, 0\right)+c^{4} f^{\Delta_{2}^{2}}\left(t_{2}\right) h_{2}\left(t_{1}, 0\right)
\end{aligned}
$$

and

$$
u_{k}\left(t_{1}, t_{2}\right)=\sum_{j=0}^{k} c^{2 j} f^{\Delta_{2}^{j}}\left(t_{2}\right) h_{j}\left(t_{1}, 0\right) .
$$

As $k$ is large enough such that $f^{\Delta_{2}^{k}}$ equals to zero, the series solution $u_{k}$ is the exact solution of (1).

Example 1 Consider the initial value problem

$$
\left\{\begin{array}{l}
u^{\Delta_{1}}=c^{2} u^{\Delta_{2}} \quad \text { on } \mathbb{T}_{1} \times \mathbb{T}_{2} \\
u\left(0, t_{2}\right)=g_{k}\left(t_{2}, 0\right) \quad \text { on } \mathbb{T}_{2}
\end{array}\right.
$$

where $g_{k}\left(t_{2}, 0\right)$ is a generalized polynomial on $\mathbb{T}_{2}$. The function

$$
u_{k}\left(t_{1}, t_{2}\right)=\sum_{j=0}^{k} c^{2 j} g_{k-j}\left(t_{2}, 0\right) h_{j}\left(t_{1}, 0\right)
$$

is the exact solution of (3). 
Proof We now verify that the obtained function $u_{k}$ actually solves the initial value problem (3). First, we show that the obtained function satisfies the initial condition. Since $h_{0}(t, s) \equiv$ 1 for all $t, s$ and $h_{j}(0,0) \equiv 1$ for $j>0$, we have

$$
u_{k}\left(0, t_{2}\right)=g_{k}\left(t_{2}, 0\right)
$$

Second, we display the obtained function $u_{k}$ satisfying the equation by

$$
\begin{aligned}
& u_{k}^{\Delta_{1}}\left(t_{1}, t_{2}\right)=\sum_{j=1}^{k} c^{2 j} g_{k-j}\left(t_{2}, 0\right) h_{j-1}\left(t_{1}, 0\right), \\
& c^{2} u_{k}^{\Delta_{2}}\left(t_{1}, t_{2}\right)=\sum_{j=0}^{k-1} c^{2(j+1)} g_{k-j-1}\left(t_{2}, 0\right) h_{j}\left(t_{1}, 0\right)=\sum_{j=1}^{k} c^{2 j} g_{k-j}\left(t_{2}, 0\right) h_{j-1}\left(t_{1}, 0\right) .
\end{aligned}
$$

This implies that $u_{k}^{\Delta_{1}}\left(t_{1}, t_{2}\right)-c^{2} u_{k}^{\Delta_{2}}\left(t_{1}, t_{2}\right)=0$ on $\mathbb{T}_{1} \times \mathbb{T}_{2}$.

\subsection{The exact solution of the second-order linear partial dynamic equations}

Consider the second-order partial dynamic equation as the form

$$
\left\{\begin{array}{l}
u^{\Delta_{1}}=c^{2} u^{\Delta_{2}^{2}} \quad \text { on } \mathbb{T}_{1} \times \mathbb{T}_{2} \\
u\left(0, t_{2}\right)=f\left(t_{2}\right) \text { on } \mathbb{T}_{2},
\end{array}\right.
$$

where $f\left(t_{2}\right)=\sum_{i=0}^{K} a_{i} g_{i}\left(t_{2}, 0\right)$ on $\mathbb{T}_{2}$ and $a_{i}, i=1, \ldots, K$ are real numbers.

In this work, the linear operator $L$ is selected as

$$
L u=u^{\Delta_{1}}
$$

and the other operator $N$ is selected as

$$
N u=-c^{2} u^{\Delta_{2}^{2}}
$$

Using the initial condition $u_{0}\left(t_{1}, t_{2}\right)=f\left(t_{2}\right)$ and the iteration formula (2), we have the following equations:

$$
\begin{aligned}
u_{1}\left(t_{1}, t_{2}\right) & =f\left(t_{2}\right)-\int_{0}^{t_{1}} c^{2} f^{\Delta_{2}^{2}}\left(t_{2}\right) \Delta s \\
& =f\left(t_{2}\right)+c^{2} f^{\Delta_{2}^{2}}\left(t_{2}\right) h_{1}\left(t_{1}, 0\right), \\
u_{2}\left(t_{1}, t_{2}\right) & =u_{1}\left(t_{1}, t_{2}\right)-\int_{0}^{t_{1}} c^{2} f^{\Delta_{2}^{4}}\left(t_{2}\right) h_{1}(s, 0) \Delta s \\
& =u_{1}\left(t_{1}, t_{2}\right)+f^{\Delta_{2}^{4}}\left(t_{2}\right) h_{2}\left(t_{1}, 0\right) \\
& =f\left(t_{2}\right)+c^{2} f^{\Delta_{2}^{2}}\left(t_{2}\right) h_{1}\left(t_{1}, 0\right)+c^{4} f^{\Delta_{2}^{4}}\left(t_{2}\right) h_{2}\left(t_{1}, 0\right)
\end{aligned}
$$

and

$$
u_{k}\left(t_{1}, t_{2}\right)=\sum_{j=0}^{k} c^{2 j} f^{\Delta_{2}^{(2 j)}}\left(t_{2}\right) h_{j}\left(t_{1}, 0\right)
$$


As $k$ is large enough such that $f^{\Delta_{2}^{(2 k)}}$ equals to zero, the series solution $u_{k}$ is the exact solution of (4).

Example 2 Consider the IVP

$$
\left\{\begin{array}{l}
u^{\Delta_{1}}=c^{2} u^{\Delta_{2}^{2}} \quad \text { on } \mathbb{T}_{1} \times \mathbb{T}_{2}, \\
u\left(0, t_{2}\right)=g_{k}\left(t_{2}, 0\right) \quad \text { on } \mathbb{T}_{2},
\end{array}\right.
$$

where $g_{k}\left(t_{2}, 0\right)$ is a generalized polynomial of $\mathbb{T}_{2}$. The function

$$
u_{\lfloor k / 2\rfloor}\left(t_{1}, t_{2}\right)=\sum_{j=0}^{\lfloor k / 2\rfloor} c^{2 j} g_{k-2 j}\left(t_{2}, 0\right) h_{j}\left(t_{1}, 0\right)
$$

is the exact solution of (5).

The exact solution of Example 2 is also obtained by Jackson [6]. He transformed the IVP into an ODE and obtained the exact solution as

$$
u\left(t_{1}, t_{2}\right)=\sum_{j=0}^{\lfloor k / 2\rfloor} c^{2 j} g_{k-2 j}\left(t_{2}, 0\right) h_{j}\left(t_{1}, 0\right),
$$

where $\lfloor k / 2\rfloor$ denotes the floor of $k / 2$.

When the initial condition can be represented as a finite series of generalized polynomials, we have proposed a useful method of finding the exact solution of partial dynamic equations on time scales. When the initial condition is represented as an infinite series of generalized polynomials, the approximate solution can be obtained by the same manner. In the following section, we consider the nonlinear partial dynamic equation on the specific time scales.

\section{Approximation solutions of nonlinear $q$-partial dynamic equations}

In this section, we extend the variational iteration method to find an approximate solution of nonlinear initial value problems on the time scale $\overline{q^{\mathbb{N}}}$.

To extend the variational iteration method, we first display a production rule [9] of two $q$-polynomials at 0 which will be used to derive an approximate solution in the following discussion.

Theorem 1 Let $h_{i}(t, 0)$ and $h_{j}(t, 0)$ be two q-polynomials at zero. We have

$$
h_{i}(t, 0) h_{j}(t, 0)=\frac{\left(q^{i+1} ; q\right)_{j}}{(q ; q)_{j}} h_{i+j}(t, 0) .
$$

Proof Since

$$
h_{i+j}(t, 0)=\prod_{\nu=0}^{i+j-1} \frac{t}{\sum_{\mu=0}^{v} q^{\mu}},
$$


we have

$$
\begin{aligned}
h_{i+j}(t, 0) & =\left(\prod_{\nu=0}^{i-1} \frac{t}{\sum_{\mu=0}^{v} q^{\mu}}\right)\left(\prod_{\nu=i}^{i+j-1} \frac{t}{\sum_{\mu=0}^{v} q^{\mu}}\right) \\
& =h_{i}(t, 0)\left(\frac{\prod_{\nu=0}^{j-1} \sum_{\mu=0}^{v} q^{\mu}}{\prod_{\nu=0}^{j-1} \sum_{\mu=0}^{v} q^{\mu}}\right) t^{j}\left(\prod_{\nu=i}^{i+j-1} \frac{1}{\sum_{\mu=0}^{v} q^{\mu}}\right) \\
& =h_{i}(t, 0)\left(\prod_{\nu=0}^{j-1} \frac{t}{\sum_{\mu=0}^{v} q^{\mu}}\right)\left(\prod_{\nu=0}^{j-1} \sum_{\mu=0}^{\nu} q^{\mu}\right)\left(\prod_{\nu=i}^{i+j-1} \frac{1}{\sum_{\mu=0}^{v} q^{\mu}}\right) \\
& =h_{i}(t, 0) h_{j}(t, 0)\left(\prod_{\nu=0}^{j-1} \frac{\sum_{\mu=0}^{v} q^{\mu}}{\sum_{\mu=0}^{v+i} q^{\mu}}\right) .
\end{aligned}
$$

This implies that

$$
\begin{aligned}
h_{i}(t, 0) h_{j}(t, 0) & =\left(\prod_{\nu=0}^{j-1} \frac{\sum_{\mu=0}^{v+i} q^{\mu}}{\sum_{\mu=0}^{v} q^{\mu}}\right) h_{i+j}(t, 0)=\prod_{\nu=0}^{j-1} \frac{\left(1-q^{v+i+1}\right)}{\left(1-q^{v+1}\right)} h_{i+j}(t) \\
& =\frac{\left(q^{i+1} ; q\right)_{j}}{(q ; q)_{j}} h_{i+j}(t, 0) .
\end{aligned}
$$

Proposition 1 Let $h_{i}(t, 0)$ and $h_{j}(t, 0)$ be any two q-polynomials. We have

$$
h_{i}(t, 0) h_{j}(t, 0)=h_{j}(t, 0) h_{i}(t, 0) .
$$

Proof It suffices to show that

$$
\frac{\left(q^{i+1} ; q\right)_{j}}{(q, q)_{j}}=\frac{\left(q^{j+1} ; q\right)_{i}}{(q, q)_{i}}
$$

Suppose $i>j$, we have

$$
\begin{aligned}
& \frac{\left(q^{i+1} ; q\right)_{j}}{(q, q)_{j}}-\frac{\left(q^{j+1} ; q\right)_{i}}{(q, q)_{i}} \\
& \quad=\frac{\left(1-q^{j+1}\right) \cdots\left(1-q^{i+j}\right)}{(1-q) \cdots\left(1-q^{i}\right)}-\frac{\left(1-q^{i+1}\right) \cdots\left(1-q^{i+j}\right)}{(1-q) \cdots\left(1-q^{j}\right)} \\
& \quad=\frac{\left(1-q^{j+1}\right) \cdots\left(1-q^{i+j}\right)}{(1-q) \cdots\left(1-q^{i}\right)}-\frac{\left(1-q^{i+1}\right) \cdots\left(1-q^{i+j}\right)\left(1-q^{j+1}\right) \cdots\left(1-q^{i}\right)}{(1-q) \cdots\left(1-q^{j}\right)\left(1-q^{j+1}\right) \cdots\left(1-q^{i}\right)}=0 .
\end{aligned}
$$

Let $h_{k}$ and $g_{k}$ be generalized polynomials of $\overline{q_{1}^{\mathbb{N}}}$ and $\overline{q_{2}^{\mathbb{N}}}$. The variational iteration method is now applied to find an approximate solution of the nonlinear partial dynamic equations as the form

$$
\left\{\begin{array}{l}
u^{\Delta_{1}}=N u \quad \text { on } \overline{q_{1}^{\mathbb{N}}} \times \overline{q_{2}^{\mathbb{N}}}, \\
u\left(0, t_{2}\right)=g_{k}\left(t_{2}, 0\right) \text { on } \overline{q_{2}^{\mathbb{N}}} .
\end{array}\right.
$$

When the linear operator $L$ is selected as

$$
L u=u^{\Delta_{1}}
$$


and the other operator $N$ is selected as $-N u$, the variational iteration formula is obtained as

$$
u_{n+1}\left(t_{1}, t_{2}\right)=u_{n}\left(t_{1}, t_{2}\right)-\int_{0}^{t_{1}}\left\{u_{n}^{\Delta_{1}}\left(s, t_{2}\right)-N u_{n}\left(s, t_{2}\right)\right\} \Delta s
$$

with the initial approximation

$$
u_{0}=g_{k}\left(t_{2}, 0\right)
$$

Example 3 Consider the partial dynamic equations as the form

$$
\left\{\begin{array}{l}
u^{\Delta_{1}}=u u^{\Delta_{2}} \quad \text { on } \mathbb{T}_{1} \times \mathbb{T}_{2}, \\
u\left(0, t_{2}\right)=g_{k}\left(t_{2}, 0\right) \quad \text { on } \mathbb{T}_{2}
\end{array}\right.
$$

With the variational iteration formula, we obtain the first few components of $u_{n}\left(t_{1}, t_{2}\right)$ :

$$
\begin{aligned}
u_{1}\left(t_{1}, t_{2}\right)= & u_{0}\left(t_{1}, t_{2}\right)-\int_{0}^{t_{1}}\left\{u_{0}^{\Delta_{1}}\left(s, t_{2}\right)-u_{0}\left(s, t_{2}\right) u_{0}^{\Delta_{2}}\left(s, t_{2}\right)\right\} \Delta s \\
= & g_{k}\left(t_{2}, 0\right)+\int_{0}^{t_{1}}\left[g_{k}\left(t_{2}, 0\right) g_{k-1}\left(t_{2}, 0\right)\right] \Delta s \\
= & g_{k}\left(t_{2}, 0\right)+H_{2}(k, k-1) g_{2 k-1}\left(t_{2}, 0\right) h_{1}\left(t_{1}, 0\right) \\
u_{2}\left(t_{1}, t_{2}\right)= & u_{1}\left(t_{1}, t_{2}\right)-\int_{0}^{t_{1}}\left\{u_{1}^{\Delta_{1}}\left(s, t_{2}\right)-u_{1}\left(s, t_{2}\right) u_{1}^{\Delta_{2}}\left(s, t_{2}\right)\right\} \Delta s \\
= & g_{k}\left(t_{2}, 0\right)+H_{2}(k, k-1) g_{2 k-1}\left(t_{2}, 0\right) h_{1}\left(t_{1}, 0\right) \\
& -\int_{0}^{t_{1}}\left[H_{2}(k, k-1) g_{2 k-1}\left(t_{2}, 0\right)-\left(g_{k}\left(t_{2}, 0\right)+H_{2}(k, k-1) g_{2 k-1}\left(t_{2}, 0\right) h_{1}(s, 0)\right)\right. \\
& \left.\times\left(g_{k-1}\left(t_{2}, 0\right)+H_{2}(k, k-1) g_{2 k-2}\left(t_{2}, 0\right) h_{1}(s, 0)\right)\right] \Delta s \\
= & g_{k}\left(t_{2}, 0\right)+H_{2}(k, k-1) g_{2 k-1}\left(t_{2}, 0\right) h_{1}\left(t_{1}, 0\right) \\
& +H_{2}(k, k-1) H_{2}(k, 2 k-2) g_{3 k-2}\left(t_{2}, 0\right) h_{2}\left(t_{1}, 0\right) \\
& +H_{2}(k, k-1) H_{2}(k-1,2 k-1) g_{3 k-2}\left(t_{2}, 0\right) h_{2}\left(t_{1}, 0\right) \\
& +H_{2}(k, k-1) H_{2}(k, k-1) H_{2}(2 k-1,2 k-2) g_{4 k-3}\left(t_{2}, 0\right) H_{1}(1,1) h_{3}\left(t_{1}, 0\right)
\end{aligned}
$$

where $H_{1}(k, l)=\frac{\left(q_{1}^{k+1} ; q_{1}\right)_{l}}{\left(q_{1} ; q_{1}\right) l}$ and $H_{2}(k, l)=\frac{\left(q_{2}^{k+1} ; q_{2}\right)_{l}}{\left(q_{2} ; q_{2}\right) l}$.

In the same manner, the rest of components of the iteration formula are obtained iteratively.

\subsection{Applications to the $q$-Burger equation and the Fisher equation}

\section{q-Burger equation}

First of all, we consider the $q$-Burger equation as the form

$$
\left\{\begin{array}{l}
u^{\Delta_{1}}-u u^{\Delta_{2}}-\alpha u^{\Delta_{2}^{2}}=0 \text { on } \overline{q_{1}^{\mathbb{N}}} \times \overline{q_{2}^{\mathbb{N}}}, \\
u\left(0, t_{2}\right)=g_{k}\left(t_{2}, 0\right) \text { on } \overline{q_{2}^{\mathbb{N}}} .
\end{array}\right.
$$


When the linear operator and the nonlinear operator are selected as $L u=u^{\Delta_{1}}$ and $-N u=$ $-u u^{\Delta_{2}}-\alpha u^{\Delta_{2}^{2}}$, respectively, the variational iteration formula is obtained as

$$
u_{n+1}\left(t_{1}, t_{2}\right)=u_{n}\left(t_{1}, t_{2}\right)-\int_{0}^{t_{1}}\left\{u_{n}^{\Delta_{1}}\left(s, t_{2}\right)-u_{n}\left(s, t_{2}\right) u_{n}^{\Delta_{2}}\left(s, t_{2}\right)-\alpha u_{n}^{\Delta_{2}^{2}}\left(s, t_{2}\right)\right\} \Delta s
$$

Let $G\left(t_{2}\right)=H_{2}(k, k-1) g_{2 k-1}\left(t_{2}, 0\right)+\alpha g_{k-2}\left(t_{2}, 0\right)$ and $H_{2}(k, l)=\frac{\left(q_{2}^{k+1} ; q_{2}\right)_{l}}{\left(q_{2} ; q_{2}\right)_{l}}$. With the initial condition $u_{0}\left(t_{1}, t_{2}\right) \equiv u\left(0, t_{2}\right)=g_{k}\left(t_{2}, 0\right)$, we have

$$
\begin{aligned}
u_{1}\left(t_{1}, t_{2}\right)= & u_{0}\left(t_{1}, t_{2}\right)-\int_{0}^{t_{1}}\left\{u_{0}^{\Delta_{1}}\left(s, t_{2}\right)-u_{0}\left(s, t_{2}\right) u_{0}^{\Delta_{2}}\left(s, t_{2}\right)-\alpha u_{0}^{\Delta_{2}^{2}}\left(s, t_{2}\right)\right\} \Delta s \\
= & g_{k}\left(t_{2}, 0\right)-\int_{0}^{t_{1}}\left\{-g_{k}\left(t_{2}, 0\right) g_{k-1}\left(t_{2}, 0\right)-\alpha g_{k-2}\left(t_{2}, 0\right)\right\} \Delta s \\
= & g_{k}\left(t_{2}, 0\right)+\left(H_{2}(k, k-1) g_{2 k-1}\left(t_{2}, 0\right)+\alpha g_{k-2}\left(t_{2}, 0\right)\right) h_{1}\left(t_{1}, 0\right) \\
= & g_{k}\left(t_{2}, 0\right)+G\left(t_{2}\right) h_{1}\left(t_{1}, 0\right), \\
u_{2}\left(t_{1}, t_{2}\right)= & u_{1}\left(t_{1}, t_{2}\right)-\int_{0}^{t_{1}}\left\{u_{1}^{\Delta_{1}}\left(s, t_{2}\right)-u_{1}\left(s, t_{2}\right) u_{1}^{\Delta_{2}}\left(s, t_{2}\right)-\alpha u_{1}^{\Delta 2}\left(s, t_{2}\right)\right\} \Delta s \\
= & g_{k}\left(t_{2}, 0\right)+G\left(t_{2}\right) h_{1}\left(t_{1}, 0\right) \\
& -\int_{0}^{t_{1}}\left\{G\left(t_{2}\right)-\left(g_{k}\left(t_{2}, 0\right)+G\left(t_{2}\right) h_{1}(s, 0)\right)\right. \\
& \times\left(g_{k-1}\left(t_{2}, 0\right)+g^{\Delta_{2}}\left(t_{2}\right) h_{1}(s, 0)\right) \\
& \left.-\alpha\left(g_{k-2}\left(t_{2}, 0\right)+g^{\Delta_{2}^{2}}\left(t_{2}\right) h_{1}(s, 0)\right)\right\} \Delta s \\
= & g_{k}\left(t_{2}, 0\right)+H_{2}(k, k-1) g_{2 k-1}\left(t_{2}, 0\right) h_{1}\left(t_{1}, 0\right) \\
& +G\left(t_{2}\right) g_{k-1}\left(t_{2}, 0\right) h_{2}\left(t_{1}, 0\right)+g^{\Delta_{2}}\left(t_{2}\right) g_{k}\left(t_{2}, 0\right) h_{2}\left(t_{1}, 0\right) \\
& +G\left(t_{2}\right) g^{\Delta_{2}}\left(t_{2}\right) H_{1}(1,1) h_{3}\left(t_{1}, 0\right) \\
& +\alpha g_{k-2}\left(t_{2}, 0\right) h_{1}\left(t_{1}, 0\right)+\alpha g^{\Delta_{2}^{2}}\left(t_{2}\right) h_{2}\left(t_{1}, 0\right) \\
= & g_{k}\left(t_{2}, 0\right)+\left[H_{2}(k, k-1) g_{2 k-1}\left(t_{2}, 0\right)+\alpha g_{k-2}\left(t_{2}, 0\right)\right] h_{1}\left(t_{1}, 0\right) \\
& +\left[G\left(t_{2}\right) g_{k-1}\left(t_{2}, 0\right)+g^{\Delta_{2}}\left(t_{2}\right) g_{k}\left(t_{2}, 0\right)+\alpha g^{\Delta_{2}^{2}}\left(t_{2}\right)\right] h_{2}\left(t_{1}, 0\right) \\
& +G\left(t_{2}\right) g^{\Delta_{2}}\left(t_{2}\right) H_{1}(1,1) h_{3}\left(t_{1}, 0\right) . \\
&
\end{aligned}
$$

In the same manner, the rest of components of the iteration formula are obtained iteratively.

q-Fisher equation

Secondly, we consider the $q$-Fisher equation, which is a nonlinear reaction diffusion equation, as the form

$$
\left\{\begin{array}{l}
u^{\Delta_{1}}-\alpha u^{\Delta_{2}^{2}}-\beta u(1-u)=0 \quad \text { on } \overline{q_{1}^{\mathbb{N}}} \times \overline{q_{2}^{\mathbb{N}}}, \\
u\left(0, t_{2}\right)=g_{k}\left(t_{2}, 0\right) \text { on } \overline{q_{2}^{\mathbb{N}}} .
\end{array}\right.
$$


The variational iteration formula is obtained as

$$
\begin{aligned}
u_{n+1}\left(t_{1}, t_{2}\right)= & u_{n}\left(t_{1}, t_{2}\right)-\int_{0}^{t_{1}}\left\{u_{n}^{\Delta_{1}}\left(s, t_{2}\right)-\alpha u_{n}^{\Delta_{2}^{2}}\left(s, t_{2}\right)\right. \\
& \left.-\beta u_{n}\left(s, t_{2}\right)+\beta u_{n}\left(s, t_{2}\right) u_{n}\left(s, t_{2}\right)\right\} \Delta s
\end{aligned}
$$

Let $G\left(t_{2}\right)=\alpha h_{k-2}\left(t_{2}\right)+\beta g_{k}\left(t_{2}, 0\right)-\beta H_{2}(k, k) g_{2 k}\left(t_{2}, 0\right)$ and $H_{2}(k, l)=\frac{\left(q_{2}^{k+1} ; q_{2}\right)_{l}}{\left(q_{2} ; q_{2}\right)_{l}}$. With the initial condition $u_{0}\left(t_{1}, t_{2}\right) \equiv u\left(0, t_{2}\right)=g_{k}\left(t_{2}, 0\right)$, we have

$$
\begin{aligned}
u_{1}\left(t_{1}, t_{2}\right)= & u_{0}\left(t_{1}, t_{2}\right)-\int_{0}^{t_{1}}\left\{u_{0}^{\Delta_{1}}\left(s, t_{2}\right)-\alpha u_{0}^{\Delta_{2}^{2}}\left(s, t_{2}\right)-\beta u_{0}\left(s, t_{2}\right)+\beta u_{0}\left(s, t_{2}\right) u_{0}\left(s, t_{2}\right)\right\} \Delta s \\
= & g_{k}\left(t_{2}, 0\right)+\int_{0}^{t_{1}} \alpha g_{k-2}\left(t_{2}, 0\right)+\beta g_{k}\left(t_{2}, 0\right)-\beta H_{2}(k, k) g_{2 k}\left(t_{2}, 0\right) \Delta s \\
= & g_{k}\left(t_{2}, 0\right)+\left(\alpha h_{k-2}\left(t_{2}\right)+\beta g_{k}\left(t_{2}, 0\right)-\beta H_{2}(k, k) g_{2 k}\left(t_{2}, 0\right)\right) h_{1}\left(t_{1}, 0\right) \\
= & g_{k}\left(t_{2}, 0\right)+G\left(t_{2}\right) h_{1}\left(t_{1}, 0\right), \\
u_{2}\left(t_{1}, t_{2}\right)= & u_{1}\left(t_{1}, t_{2}\right)-\int_{0}^{t_{1}}\left\{u_{1}^{\Delta_{1}}\left(s, t_{2}\right)-\alpha u_{1}^{\Delta_{2}^{2}}\left(s, t_{2}\right)-\beta u_{1}\left(s, t_{2}\right)+\beta u_{1}\left(s, t_{2}\right) u_{1}\left(s, t_{2}\right)\right\} \Delta s \\
= & g_{k}\left(t_{2}, 0\right)+G\left(t_{2}\right) h_{1}\left(t_{1}, 0\right) \\
& -\int_{0}^{t_{1}}\left\{G\left(t_{2}\right)-\alpha\left(g_{k-2}\left(t_{2}, 0\right)+g^{\Delta}\left(t_{2}\right) h_{1}(s, 0)\right)-\beta\left(g_{k}\left(t_{2}, 0\right)+G\left(t_{2}\right) h_{1}(s, 0)\right)\right. \\
& \left.+\beta\left(g_{k}\left(t_{2}, 0\right)+G\left(t_{2}\right) h_{1}(s, 0)\right)\left(g_{k}\left(t_{2}, 0\right)+G\left(t_{2}\right) h_{1}(s, 0)\right)\right\} \Delta s \\
& +\left(\alpha g^{\Delta \Delta_{2}^{2}}\left(t_{2}\right)+\beta G\left(t_{2}\right)-2 \beta G\left(t_{2}\right) g_{k}\left(t_{2}, 0\right)\right) h_{2}\left(t_{1}, 0\right) \\
& g_{k}\left(t_{2}, 0\right)+\alpha g_{k-2}^{2}\left(t_{2}, 0\right) h_{1}\left(t_{1}, 0\right)+\alpha g_{1}^{\Delta_{2}^{2}}\left(t_{2}\right) h_{2}\left(t_{1}, 0\right) \\
& +\beta g_{k}\left(t_{2}, 0\right) h_{1}\left(t_{1}, 0\right)+\beta G\left(t_{2}\right) h_{2}\left(t_{1}, 0\right) \\
& -g_{0}^{t_{1}} \beta\left(t_{2}\right) H_{1}\left(t_{1}, 0\right) . \\
= & g_{k}\left(t_{2}, 0\right)+\left[\alpha g_{k-2}\left(t_{2}, 0\right)+\beta g_{2}\left(t_{2}, 0\right)-\beta H_{2}(k, k) g_{2 k}\left(t_{2}, 0\right)\right] h_{1}\left(t_{1}, 0\right) \\
& \beta s)+2 G\left(t_{2}\right) g_{k}\left(t_{2}, 0\right) h_{1}(s, 0) \\
& \\
&
\end{aligned}
$$

In the same manner, the rest of components of the iteration formula are obtained iteratively.

\section{Numerical results}

The approximate solutions introduced in the previous sections will be illustrated with some examples.

Let $\mathbb{T}_{1}=\mathbb{T}_{2}=\overline{0.9^{\mathbb{N}}}=\{0.9,0.81,0.729, \ldots, 0\}$, where 0 is the cluster point of $\overline{q^{\mathbb{N}}}$. The $q$-shift factorial with $q=0.9$ is given as

$$
(a ; 0.9)_{0}=1 \quad \text { and } \quad(a ; 0.9)_{n}=\prod_{k=0}^{n-1}\left(1-0.9^{k} a\right), \quad n \in \mathbb{N}
$$


and the $q$-polynomials are represented as

$$
h_{k}(t, 0)=\prod_{\nu=0}^{k-1} \frac{t}{\sum_{j=0}^{v} 0.9^{j}}=\frac{t^{k}}{\prod_{\nu=0}^{k-1} \sum_{j=0}^{v} 0.9^{j}}=\frac{t^{k}}{\prod_{\nu=1}^{k-1} 9\left(1-0.9^{\nu}\right)},
$$

$t \in\left\{0.9^{n}, n \in \mathbb{N}\right\} \cup\{0\}$ and $h_{k}(0,0)=1$. The multiplication of two generalized polynomials $h_{k}(t, 0)$ and $h_{l}(t, 0)$ is obtained as

$$
h_{k}(t, 0) h_{l}(t, 0)=H(k, l) h_{k+l}(t, 0) \text {, }
$$

where $H(k, l)=\frac{\left(0.9^{k+1} ; 0.9\right)_{l}}{(0.9 ; 0.9)_{l}}$. For example, $H(1,1)=\frac{\left(0.9^{2} ; 0.9\right)_{1}}{(0.9 ; 0.9)_{1}}=\frac{\left(1-0.9^{2}\right)}{(1-0.9)}=\frac{1.9}{0.1}=1.9$.

Example 4 Consider the nonlinear partial dynamic equation as the form

$$
\left\{\begin{array}{l}
u^{\Delta_{1}}-u u^{\Delta_{2}}-u^{\Delta_{2}^{2}}=0 \text { on } \overline{0.9^{\mathbb{N}}} \times \overline{0.9^{\mathbb{N}}}, \\
u\left(0, t_{2}\right)=g_{1}\left(t_{2}, 0\right) \text { on } \overline{0.9^{\mathbb{N}}} .
\end{array}\right.
$$

The initial approximation can be given as

$$
u_{0}\left(t_{1}, t_{2}\right)=g_{1}\left(t_{2}, 0\right)
$$

according to the initial condition. By the variational iteration formula (2), the first two components of $u_{n}\left(t_{1}, t_{2}\right)$ are obtained:

$$
\begin{aligned}
& u_{1}\left(t_{1}, t_{2}\right)=\left(1+h_{1}\left(t_{1}, 0\right)\right) g_{1}\left(t_{2}, 0\right), \\
& u_{2}\left(t_{1}, t_{2}\right)=\left(1+h_{1}\left(t_{1}, 0\right)+2 h_{2}\left(t_{1}, 0\right)+1.9 h_{3}\left(t_{1}, 0\right)\right) g_{1}\left(t_{2}, 0\right) .
\end{aligned}
$$

The rest of components of the iteration formula are obtained in the same manner. The responses of $u\left(t_{1}, t_{2}\right)$ are shown in Figure 1.

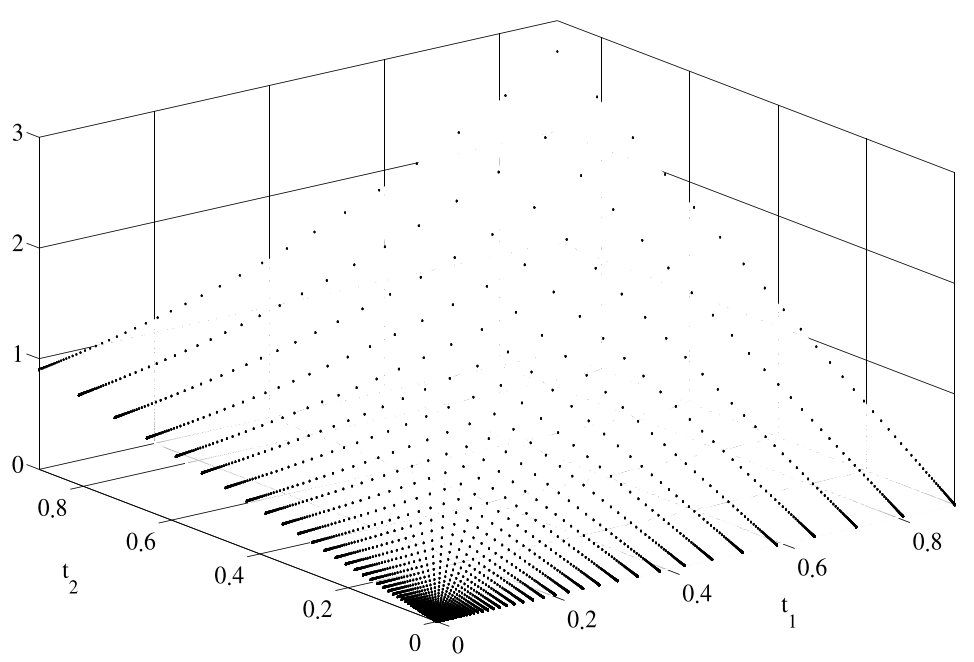

Figure 1 Response for (8) with 2 VIM iterations. 
Example 5 Consider the Fisher equation as the form

$$
\left\{\begin{array}{l}
u^{\Delta_{1}}-u^{\Delta_{2}^{2}}-u(1-u)=0 \quad \text { on } \overline{0.9^{\mathbb{N}}} \times \overline{0.9^{\mathbb{N}}} \\
u\left(0, t_{2}\right)=g_{1}\left(t_{2}, 0\right) \text { on } \overline{0.9^{\mathbb{N}}}
\end{array}\right.
$$

With the initial condition $u_{0}\left(t_{1}, t_{2}\right)=g_{1}\left(t_{2}, 0\right)$, the first two components are obtained

$$
\begin{aligned}
u_{1}\left(t_{1}, t_{2}\right)= & g_{1}\left(t_{2}, 0\right)+\left(g_{1}\left(t_{2}, 0\right)-1.9 g_{2}\left(t_{2}, 0\right)\right)_{1}\left(t_{2}, 0\right)=g_{1}\left(t_{2}, 0\right)+G\left(t_{2}\right) g_{1}\left(t_{2}, 0\right), \\
u_{2}\left(t_{1}, t_{2}\right)= & g_{1}\left(t_{2}, 0\right)+G\left(t_{2}\right) h_{1}\left(t_{1}, 0\right)+\left[g^{\Delta_{2}^{2}}\left(t_{2}\right)+G\left(t_{2}\right)-2 G\left(t_{2}\right) g_{1}\left(t_{2}, 0\right)\right] h_{2}\left(t_{1}, 0\right) \\
& -g^{2}\left(t_{2}\right) H_{1}(1,1) h_{3}\left(t_{1}, 0\right) \\
= & g_{1}\left(t_{2}, 0\right)+\left[g_{1}\left(t_{2}, 0\right)-1.9 g_{2}\left(t_{2}, 0\right)\right] h_{1}\left(t_{1}, 0\right) \\
& +\left[-1.9+g_{1}\left(t_{2}, 0\right)-1.9 g_{2}\left(t_{2}, 0\right)-2\left(g_{1}\left(t_{2}, 0\right)-1.9 g_{2}\left(t_{2}, 0\right)\right) g_{1}\left(t_{2}, 0\right)\right] h_{2}\left(t_{1}, 0\right) \\
& -1.9\left(g_{1}\left(t_{2}, 0\right)-1.9 g_{2}\left(t_{2}, 0\right)\right)^{2} h_{3}\left(t_{1}, 0\right) \\
= & g_{1}\left(t_{2}, 0\right)+\left[g_{1}\left(t_{2}, 0\right)-1.9 g_{2}\left(t_{2}, 0\right)\right] h_{1}\left(t_{1}, 0\right) \\
& +\left[-1.9+g_{1}\left(t_{2}, 0\right)-5.7 g_{2}\left(t_{2}, 0\right)+10.298 g_{3}\left(t_{2}, 0\right)\right] h_{2}\left(t_{1}, 0\right) \\
& -1.9\left[1.9 g_{2}\left(t_{2}, 0\right)-10.298 g_{3}\left(t_{2}, 0\right)+4.9051 g_{4}\left(t_{2}, 0\right)\right] h_{3}\left(t_{1}, 0\right),
\end{aligned}
$$

where $G\left(t_{2}\right)=g_{1}\left(t_{2}, 0\right)-1.9 g_{2}\left(t_{2}, 0\right)$. The rest of components of the iteration formula are obtained in the same manner. The responses of $u\left(t_{1}, t_{2}\right)$ are shown in Figure 2.

Now, we have demonstrated a method for finding an approximate solution of nonlinear partial dynamic equations on $\overline{q_{1}^{\mathbb{N}}} \times \overline{q_{2}^{\mathbb{N}}}$. The proposed tool could also be applied to other nonlinear $q$-partial dynamic equations.

In future studies, we intend to derive the multiplication rule of two generalized polynomials and extend the application of the variational iteration method to nonlinear partial dynamic equations on other time scales.

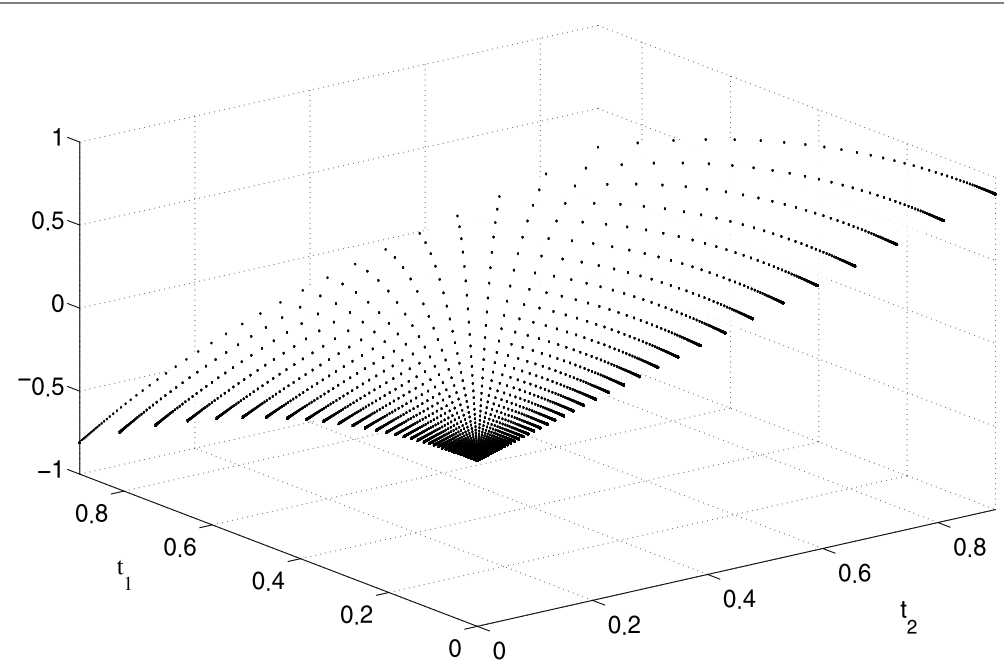

Figure 2 Response for (9) with 2 VIM iterations. 


\section{Conclusion and future direction}

In this paper, we have propose a method to find the exact solution of the linear partial dynamic equation on time scales and to find an approximate solution of the nonlinear $q$-partial dynamic equations. Moreover, this method is applied to provide an approximate solution of the $q$-Berger equations and the $q$-Fisher equations.

To extend the method to other time scales, it is important to derive a multiplication rule of two generalized polynomials on the other time scales. On the other hand, approximate solutions as well as their properties of the nonlinear partial dynamic equations, such as Benjamin-Ono equations and the Benjamin-Bona-Mahony equations, are not found on $\overline{q^{\mathbb{N}}}$ yet. In the future studies, we would intend to derive the multiplication rule of two generalized polynomials or to provide an approximation of other nonlinear $q$-partial dynamic equations by using the proposing method.

\section{Appendix: Basic ideas of the variational iteration method}

To clarify the ideas of the variational iteration method, we consider the following nonlinear equation:

$$
L u(t)+N u(t)=g(t),
$$

where $L$ is a linear operator, $N$ is a nonlinear operator and $g$ is an inhomogeneous term. According to the variational iteration method, we can construct a correction functional as follows:

$$
u_{n+1}(t)=u_{n}(t)+\int_{0}^{t} \lambda\left\{L u_{n}(s)+N \tilde{u}_{n}(s)-g(s)\right\} d s
$$

where $\lambda$ is a general Lagrange multiplier, $u_{0}$ is an initial approximation which must be chosen suitably and $\tilde{u}_{n}$ is considered as a restricted variation, that is, $\delta \tilde{u}_{n}=0$. To find the optimal value of $\lambda$, we make the above correction functional stationary with respect to $u_{n}$, noticing that $\delta u_{n}(0)=0$, and have

$$
\delta u_{n+1}(t)=\delta u_{n}(t)+\delta \int_{0}^{t} \lambda L u_{n}(s) d s=0 .
$$

Having obtained the optimal Lagrange multiplier, the successive approximations $u_{n}, n \geq 0$, of the solution $u$ are determined upon the initial function $u_{0}$. Therefore, the exact solution is obtained at the limit of the resulting successive approximations.

\section{Competing interests}

The author declares that they have no competing interests.

Received: 14 November 2012 Accepted: 30 April 2013 Published: 17 May 2013

References

1. Bohner, M, Peterson, A: Dynamic Equations on Time Scales: An Introduction with Applications. Birkhäuser, Boston (2001)

2. Hoffacker, J: Basic partial dynamic equations on time scales. J. Differ. Equ. Appl. 8, 307-319 (2002) (in honor of Professor Lynn Erbe)

3. Ahlbrandt, CD, Morian, C: Partial differential equations on time scales. J. Comput. Appl. Math. 141, $35-55$ (2002)

4. Bohner, M, Guseinov, GS: Partial differentiation on time scales. Dyn. Syst. Appl. 13, 351-379 (2004)

5. Bohner, M, Guseinov, GS: Multiple integration on time scales. Dyn. Syst. Appl. 14, 579-606 (2005) 
6. Jackson, B: Partial dynamic equations on time scales. J. Comput. Appl. Math. 186, 391-415 (2006)

7. Haile, BD, Hall, LM: Polynomial and series solutions of dynamic equations on time scales. Dyn. Syst. Appl. 12, 149-157 (2003)

8. Mozyrska, D, Pawtuszewicz, E: Hermite's equations on time scales. Appl. Math. Lett. 22, 1217-1219 (2009)

9. Liu, H-K: The formula for the multiplicity of two generalized polynomials on the time scale. Appl. Math. Lett. 25, 1420-1425 (2012)

10. He, J-H: Variational iteration method - some recent results and new interpretations. J. Comput. Appl. Math. 207, 3-17 (2007)

11. Momani, S, Abusasd, S, Odibat, Z: Variational iteration method for solving nonlinear boundary value problems. Appl. Math. Comput. 183, 1351-1358 (2006)

12. Ganji, DD, Afrouzi, GA, Talarposhti, RA: Application of variational iteration method and homotopy-perturbation method for nonlinear heat diffusion and heat transfer equations. Phys. Lett. A 368, 450-457 (2007)

13. Ganji, DD, Afrouzi, GA, Talarposhtib, RA: Application of He's variational iteration method for solving the reaction-diffusion equation with ecological parameters. Comput. Math. Appl. 54, 1010-1017 (2007)

14. Hilger, S: Analysis on measure chains - a unified approach to continuous and discrete calculus. Results Math. 18 18-56 (1990)

15. Koornwinder, TH: q-special functions: a tutorial. arXiv:math/9403216v1

doi:10.1186/1687-1847-2013-141

Cite this article as: Liu: The method of finding solutions of partial dynamic equations on time scales. Advances in Difference Equations 2013 2013:141.

\section{Submit your manuscript to a SpringerOpen ${ }^{\ominus}$ journal and benefit from:}

- Convenient online submission

- Rigorous peer review

- Immediate publication on acceptance

- Open access: articles freely available online

- High visibility within the field

- Retaining the copyright to your article 